\title{
On using a too large integration time step in molecular dynamics simulations of coarse-grained molecular models
}

\author{
Received 22nd October 2008, Accepted 28th January 2009 \\ First published as an Advance Article on the web 18th February 2009 \\ DOI: $10.1039 / b 818713 d$
}

Moritz Winger, Daniel Trzesniak, Riccardo Baron and Wilfred F. van Gunsteren*

\begin{abstract}
The use of a coarse-grained (CG) model that is widely used in molecular dynamics simulations of biomolecular systems is investigated with respect to the dependence of a variety of quantities upon the size of the used integration time step and cutoff radius. The results suggest that when using a non-bonded interaction-cutoff radius of $1.4 \mathrm{~nm}$ a time step of maximally $10 \mathrm{fs}$ should be used, in order not to produce energy sinks or wells. Using a too-large time step, e.g. $50 \mathrm{fs}$ with a cutoff of $1.2 \mathrm{~nm}$, as is done in the coarse-grained model of Marrink et al. (J. Phys. Chem. B, 2004, 108, 250 and 2007, 111, 7812), induces errors due to the linear approximation of the integrators that are commonly used to integrate the equations of motion. As a spin-off of the investigation of the mentioned CG models, we found that the parameters of the CG water model place it at physiological temperatures well into the solid phase of the phase diagram.
\end{abstract}

\section{Introduction}

Coarse-grained (CG) models for molecular simulation, in which groups of atoms are modelled as one particle or coarse-grained atom, are very popular these days. ${ }^{1,2}$ Their greatest advantage is the reduction of computational power required, since fewer interaction sites have to be taken into account. This makes simulations of larger systems at longer timescales feasible. Different CG models have been proposed, see for example ref. 3-18. A much used one for lipids and water is the one developed by Marrink et al. ${ }^{10,14}$ which has been extended to proteins. ${ }^{15}$ In some applications ${ }^{19,20}$ and $\mathrm{CG}$ model parametrisations ${ }^{10,14,15}$ rather large time steps $\Delta t$ of $30-50 \mathrm{fs}$ are used in the numerical propagation of the solutions of the Newtonian equations of motion. These values are a factor 20 larger than the values of $0.5-2.0 \mathrm{fs}$ used for all-atom models. ${ }^{21,22}$ The use of large time steps in CG models is justified by invoking the smoother energy function or surface compared to all-atom, or fine-grained (FG), model functions. However, a $\Delta t$ difference of a factor 20 seems too large considering that only about 4 non-hydrogen real atoms are mapped onto one CG atom in the most common CG model for lipids and water. ${ }^{10,14}$

Since, in MD, Newton's equations of motion are integrated forward in time using discrete time steps $\Delta t$ and truncated Taylor series expansions of the positions $r(t)$ and velocities $v(t)$ of the atoms at the succeeding time points $t_{n}$, the maximum size of the time step $\Delta t$ is restricted by the size of the (higher-order) time derivatives of $r$ and $v$ that are omitted from the Taylor expansions in the particular integration algorithm used. The

Laboratory of Physical Chemistry, Swiss Federal Institute of

Technology, ETH, 8093 Zürich, Switzerland.

E-mail:wfvgn@igc.phys.chem.ethz.ch.

E-mail: igc-sec@igc.phys.chem.ethz.ch; Fax: +41(0)446321039;

Tel: $+41(0) 446325501$ time derivatives of $r(t)$ and $v(t)$ involve derivatives of the forces $f(t)$ with respect to time, because the acceleration $a(t)$ of an atom equals the force on it divided by its mass. The change of the force with time, therefore, depends on the ruggedness of the atomic or molecular interaction function $V(r)$ : the smoother it is, the smaller the change in $f$ with distance $\Delta r$ covered will be.

Thirty years ago, the time step, $\Delta t$, that is compatible with a reasonably accurate integration of the equations of motion for typical all-atom biomolecular interaction functions was analyzed and found to be $0.5-2$ fs. $^{21-23}$ Since the CG model of ref. 10 and 14 involves the same functional form for the interaction function, a $r^{-12}, r^{-6}$, and $r^{-1}$ distance-dependence of the non-bonded interaction, it is unlikely that it can be used with a ten or more times longer time step $\Delta t$. Here, we investigate this issue and show that, using a typical CG model, time steps larger than 10 fs lead to very poor integration of the equations of motion, resulting in average values of properties, e.g. temperatures, that are different from the correct ones. Taking a widely used CG model ${ }^{10,14}$ as example, we analyze for systems consisting of alkanes and of water their properties as a function of the size of $\Delta t$, of the size of the cut-off radius $R_{\mathrm{c}}$ for non-bonded interactions and of the strength of the coupling of the system to a heat bath to stabilize the temperature. The properties are thermodynamic: (density, heat of vaporisation, excess free energy, conformational entropy (alkanes) and solvation free energy); dynamic: diffusion; and structural: (radial distribution functions and for alkanes, bond-angle, torsional-angle and end-to-end of chain distance distributions).

During our investigation of the time step issue, in which we used the CG water model of ref. 10 and 14, we found that this model constitutes a poor approximation of liquid water, because its parameters are such that at physiological temperatures the model is well into the solid part of its phase diagram. 


\section{Computational methods}

The coarse-grained (CG) model of Marrink et al. ${ }^{10,14,15}$ has been formulated in terms of a Lennard-Jones function which is modified by using a particular shift function as implemented in the GROMACS ${ }^{24}$ molecular simulation program. Since this shift function was erroneously described in the GROMACS manual and also erroneously implemented in the GROMACS program, as has been extensively investigated and reported in ref. 25, we did not want to use exactly the same (inconsistent) shift function, but the shift function as implemented in the GROMOS05 biomolecular simulation software. ${ }^{26}$ To this end we investigated for which values of the parameters $R_{\mathrm{s}}$ and $R_{\mathrm{c}}$, the distances between which the non-bonded interaction is shifted, the GROMOS05 shift function would mimic the effect of the GROMACS one best. ${ }^{25}$ The original CG model of Marrink $^{10,14,15}$ has been parametrized using $R_{\mathrm{s}}=0.9 \mathrm{~nm}$, $R_{\mathrm{c}}=1.2 \mathrm{~nm}$ and the (inconsistent) GROMACS shift function. Using $R_{\mathrm{s}}=0 \mathrm{~nm}$ and $R_{\mathrm{c}}=1.4 \mathrm{~nm}$ and the GROMOS05 shifting function the non-bonded interaction energy function is virtually identical to the one using GROMACS and Marrink's parameters, see Fig. 6 of ref. 25. Thus we use GROMOS05 shifting with $R_{\mathrm{s}}=0 \mathrm{~nm}$ and $R_{\mathrm{c}}=1.4 \mathrm{~nm}$ when evaluating the CG model of ref. 10, 14 and 15 .

The non-bonded interaction parameters for water $(\mathrm{W})$ and carbon $(\mathrm{C})$ coarse-grained atoms are $\mathrm{C}_{12}(\mathrm{~W}, \mathrm{~W})=2.324 \times$ $10^{-3} \mathrm{~kJ} \mathrm{~mol}^{-1} \mathrm{~nm}^{12}, \mathrm{C}_{6}(\mathrm{~W}, \mathrm{~W})=0.2156 \mathrm{~kJ} \mathrm{~mol}^{-1} \mathrm{~nm}^{6}$, $\mathrm{C}_{12}(\mathrm{C}, \mathrm{W})=0.8366 \times 10^{-3} \mathrm{~kJ} \mathrm{~mol}^{-1} \mathrm{~nm}^{12}, \mathrm{C}_{6}(\mathrm{C}, \mathrm{W})=$ $0.07761 \mathrm{~kJ} \mathrm{~mol}^{-1} \mathrm{~nm}^{6}$, and $\mathrm{C}_{12}(\mathrm{C}, \mathrm{C})=1.580 \times$ $10^{-3} \mathrm{~kJ} \mathrm{~mol}^{-1} \mathrm{~nm}^{12}, \mathrm{C}_{6}(\mathrm{C}, \mathrm{C})=0.1466 \mathrm{~kJ} \mathrm{~mol}^{-1} \mathrm{~nm}^{6}$. The bonded energy terms were as in ref. 10 and 14 . The bond- and bond-angle motions were modelled using harmonic functions: $K_{\mathrm{b}}=1250 \mathrm{~kJ} \mathrm{~mol}^{-1} \mathrm{~nm}^{-2}$, and $K_{\theta}=25 \mathrm{~kJ} \mathrm{~mol}^{-1}$, and $b_{0}=0.47 \mathrm{~nm}$ and $\theta_{0}=180^{\circ} .{ }^{10}$ No torsional potential energy term was used. ${ }^{10}$ The atomic masses were $72 \mathrm{amu}$ for CG carbon and 72 amu for CG water.

The equations of motion were integrated using the standard leap-frog algorithm, which generally only uses the first two terms on the right hand side of the equations ${ }^{27}$

$$
\begin{gathered}
v\left(t_{n}+\Delta t / 2\right)=v\left(t_{n}-\Delta t / 2\right)+m^{-1} f\left(t_{n}\right) \Delta t \\
+\frac{1}{24} m^{-1} \ddot{f}\left(t_{n}\right)(\Delta t)^{3}+O\left((\Delta t)^{5}\right) \\
r\left(t_{n}+\Delta t\right)=r\left(t_{n}\right)+v\left(t_{n}+\Delta t / 2\right) \Delta t \\
+\frac{1}{24} m^{-1} \dot{f}\left(t_{n}+\Delta t / 2\right)(\Delta t)^{3}+O\left((\Delta t)^{5}\right)
\end{gathered}
$$

where the time derivative of a quantity $f$ is denoted as $\dot{f}$. The non-bonded interaction pairlist was updated every 5 steps. Periodic boundary conditions in a cubic box were applied. If the temperature was to be held constant, this was done by weak coupling to a temperature bath $\left(T_{\text {ref }}=298 \mathrm{~K}\right)$ with relaxation times $\tau_{T}=0.1 \mathrm{ps}, 1 \mathrm{ps}$ or $10 \mathrm{ps} .{ }^{28}$ For water the box volume was $343 \mathrm{~nm}^{3}$ and for hexadecane $216 \mathrm{~nm}^{3}$. In the simulations with constant pressure the systems were in addition weakly coupled to a pressure bath ${ }^{28}$ of 1 atm with a
Table 1 Overview of the performed MD simulations on coarsegrained water and coarse-grained alkanes

\begin{tabular}{lll}
$\begin{array}{l}\text { Number of } \\
\text { molecules }\end{array}$ & System & Property held constant \\
\hline Water & Hexadecane & \\
3200 & 0 & NVE, NVT, NPT \\
0 & 343 & NVE, NVT, NPT \\
1192 & 1 & NPT \\
\hline
\end{tabular}

relaxation time of $\tau_{p}=5 \tau_{T}$ and an isothermal compressibility of $0.4575 \times 10^{-3}\left(\mathrm{~kJ} \mathrm{~mol}^{-1} \mathrm{~nm}^{-3}\right)^{-1}$. Different systems were investigated: pure $\mathrm{CG}$ water, pure $\mathrm{CG}$ hexadecane $\left(\mathrm{C}_{16}\right)$, and $1 \mathrm{CG}$ hexadecane molecule in $\mathrm{CG}$ water, see Table 1 . The $\mathrm{CG}$ simulations of liquid water and liquid hexadecane were carried out with constant volume and energy (NVE), at constant volume and temperature (NVT), and at constant temperature and pressure (NPT), the solution of hexadecane in water only at constant temperature and pressure (NPT). The lengths of the simulations were 1260 ps (NVE) and 1890 ps (NPT).

Thermodynamic integration was used to compute the excess free energies of the liquids and the free enthalpies of solvation using the procedure described in ref. 25. Twenty-one equidistant values of the coupling parameter $\lambda$ were used with 63-ps equilibration and 630-ps sampling for each $\lambda$ value.

\section{Analysis}

The simulations with constant energy (NVE) were performed to evaluate how well the total energy $\left(E_{\text {tot }}\right)$ was conserved. To this end, the fluctuations, defined as $\Delta E=\left\langle[E-\langle E\rangle]^{2}\right\rangle^{1 / 2}$, of the total energy $\left(E_{\mathrm{tot}}\right)$ were compared to those of the potential $\left(E_{\text {pot }}\right)$, and kinetic energy $\left(E_{\text {kin }}\right)$. Simulating at constant total energy (NVE), $\Delta E_{\text {tot }}$ should be significantly smaller than $\Delta E_{\text {kin. }}{ }^{21,23}$ If the total energy fluctuations are not equal to zero (or very small), energy sinks or sources are present in the simulation. These can have a number of different origins: finite machine precision of the computer used, time integration errors due to truncation of higher-order (in $\Delta t$ ) terms in the (leap-frog) algorithm, use of spatial (bond-length) constraints formulated in terms of Lagrange multipliers, ${ }^{21,29}$ use of a non-bonded interaction cut-off radius, temperature-bath coupling, pressure-bath coupling, and use of non-conservative forces, such as in Langevin dynamics or when changing forcefield parameters as a function of time as in local-elevation $\mathrm{MD}^{30}$ or when applying time-averaged restraining in structure refinement of biomolecules. ${ }^{31}$ One may estimate the heat flow $\Delta Q$ into the system induced by the mentioned sinks or sources using the following expressions.

To estimate the integration error in the standard leap-frog scheme, one may evaluate the next-order corrections to the positions and velocities: ${ }^{27}$

$$
\Delta r_{n+1}=\frac{1}{24} m^{-1} \dot{f}_{n+1 / 2}(\Delta t)^{3}=\frac{1}{24 m}\left(f_{n+1}-f_{n}\right)(\Delta t)^{2}
$$

and

$$
\Delta v_{n+\frac{1}{2}}=\frac{1}{24} m^{-1} \ddot{f}_{n}(\Delta t)^{3}=\frac{1}{24 m}\left(f_{n+1}-2 f_{n}+f_{n-1}\right)(\Delta t)
$$

where we have used the short-hand notation $f_{n}$ for $f\left(t_{n}\right)$. 
Use of constraints (SHAKE) ${ }^{29}$ will also change the energy of the system because of numerical resetting of the coordinates from the unconstrained positions $r_{n+1}^{n c}$ to the (constrained) positions $r_{n+1}$. The constraint force $f_{n}^{\mathrm{c}}$ is

$$
f_{n}^{\mathrm{e}}=m\left(r_{n+1}-r_{n+1}^{\mathrm{nc}}\right) /(\Delta t)^{2}
$$

and the work done becomes

$$
\Delta Q=f_{n}^{\mathrm{c}}\left(r_{n+1}-r_{n}\right)
$$

The energy change due to the use of a cut-off radius is

$$
\begin{aligned}
\Delta Q= & -E_{\text {pot }} \text { (atoms leaving the cut-off sphere) } \\
& +E_{\text {pot }}(\text { atoms entering the cut-off sphere). }
\end{aligned}
$$

In constant temperature simulations, the thermostat will supply and take energy from the system. Using weak coupling to a temperature bath of temperature $T_{\text {ref }}$, as described in ref. 28, we find

$$
\begin{aligned}
\Delta Q & =E_{\mathrm{kin}}(\text { after velocity scaling })-E_{\mathrm{kin}}(\text { before velocity scaling }) \\
& =\sum_{i=1}^{N} \frac{1}{2} m_{i} v_{i}^{2}\left[\lambda_{s c}^{2}-1\right]
\end{aligned}
$$

with

$$
\lambda_{\mathrm{sc}}=\left[1+\frac{2 c_{v}^{\mathrm{df}}}{k_{\mathrm{B}}} \frac{\Delta t}{\tau_{T}}\left[\frac{T_{\mathrm{ref}}}{T(t-\Delta t / 2)}-1\right]\right]^{1 / 2},
$$

where $c_{v}^{\mathrm{df}}$ is the heat capacity per degree of freedom and $k_{\mathrm{B}}$ Boltzmann's constant. In practice the factor $2 c_{v}^{\mathrm{df}} / k_{\mathrm{B}}$ is set to $1 .^{28}$

When simulating at constant pressure $P_{\text {ref }}$ energy flow is also induced by the barostat. In that case we have using weak coupling $^{28}$

$$
\Delta Q=P_{\text {ref }} V\left[\mu_{\mathrm{sc}}^{3}-1\right]
$$

with

$$
\mu_{\mathrm{sc}}=\left[1-\frac{\beta_{\tau} \Delta t}{\tau_{p}}\left[P_{\mathrm{ref}}-P(t)\right]\right]^{1 / 3},
$$

where $\beta_{\tau}$ is the isothermal compressibility of the system and $V$ its volume. If the scaling factors $\lambda_{\mathrm{sc}}$ or $\mu_{\mathrm{sc}}$ are not equal to one, energy sources or sinks are present.

For the systems consisting of pure liquids, coarse-grained hexadecane and water, energy fluctuations, the higher-order terms of the leap-frog integrator algorithm, average temperature, average volume, average potential energy and the average of the squared temperature scaling factor $\lambda_{\mathrm{sc}}$ and the average of the cubed pressure scaling factor $\mu_{\mathrm{sc}}$ have been calculated. Additionally properties such as excess free energy, for both water and hexadecane, and angle distributions, dihedral angle distributions, and end-to-end of chain distances for hexadecane have been calculated. ${ }^{32}$ The free enthalpy of solvation of hexane in water was computed too.

\section{Results}

Fig. 1 and 2 show the results for the simulations of water and hexadecane at constant volume and energy. If we use, as a criterion for a not too-inaccurate integration of the equations of motion, that the fluctuations of the total energy should be less than one fifth of the fluctuations of the kinetic or potential energies, ${ }^{21-23}$ we see that it is not fulfilled using time steps larger than $5 \mathrm{fs}$ for a cutoff radius $R_{\mathrm{c}}=0.8 \mathrm{~nm}$, and using time steps larger than $10 \mathrm{fs}$ for a cutoff radius $R_{\mathrm{c}}=1.4 \mathrm{~nm}$. As expected, using a larger cutoff radius the higher-order terms (3) and (4) in the leap-frog scheme become relatively more significant when integrating the equations of motion. We also observe that using the same time step and cut-off radius the total energy conservation is better for hexadecane than for water, which is due to the stronger non-bonded interaction of water. In order to be able to use the standard leap-frog scheme, a time step of $10 \mathrm{fs}$ or smaller has to be used at a cutoff radius of $1.4 \mathrm{~nm}$. We note that for the $\mathrm{CG}$ water and CG alkane models of ref. 10 and 14 a time step of $10 \mathrm{fs}$ is equivalent to time steps of 0.0056 (water) and 0.0047 (alkanes) in reduced units. This also shows that a 50 fs time step is unacceptably long.

When coupling the system to a heat bath and a pressure bath (NPT), the thermostat and barostat will supply or withdraw energy from the system in order to keep it at the reference temperature $T_{\text {ref }}$ and reference pressure $P_{\text {ref. }}$ Fig. 3 and 4 show this effect for liquid water and liquid hexadecane, respectively. The first row panels of Fig. 3 and 4 show that beyond a time step of $20 \mathrm{fs}$, the reference temperature (298 K) is not conserved any more, because the thermostat cannot keep up with the energy loss of the system due to integration errors leading to cooling of the system by up to $20 \mathrm{~K}$. The scaling factors $\lambda_{\mathrm{sc}}^{2}(9)$ and $\mu_{\mathrm{sc}}^{3}$ (11) show deviations from the ideal value 1 at larger time steps (rows 2 and 3). The results suggest that using a cut-off radius of $1.2 \mathrm{~nm}$ or $1.4 \mathrm{~nm}$, the time step should be smaller than $20 \mathrm{fs}$.

Physical-chemical properties such as average temperature, density, potential energy, excess free energy and diffusion constant are shown in Table 2 for selected settings of cutoff radius and time step. Most properties are significantly affected by the use of time steps beyond $10 \mathrm{fs}$. Results indicate that for $R_{\mathrm{c}}=1.2 \mathrm{~nm}$ or $1.4 \mathrm{~nm}$ the time step should not be longer than $10 \mathrm{fs}$.

In Fig. 5 the radial distribution functions $g(r)$ are shown for the 1890 ps long simulations of the pure liquids (NPT) for five different combinations of the cutoff radius $R_{\mathrm{c}}$ and time step $\Delta t$. The structure of the liquids is little-affected by the use of too-large time steps. We note that the $g(r)$ for $\mathrm{CG}$ water indicates that the liquid is frozen. At $298 \mathrm{~K}$, the system of $N=3200 \mathrm{CG}$ water particles with $\varepsilon=5.0 \mathrm{~kJ} \mathrm{~mol}^{-1}$, $\sigma=0.47 \mathrm{~nm}$ and a volume $V=343 \mathrm{~nm}^{3}$ has a reduced density $\rho^{*}=0.97$ and a reduced temperature $T^{*}=0.50$, for which it is well into the solid state region of the known phase diagram of a Lennard-Jones system. ${ }^{33}$ This freezing of the CG model of water has been observed before ${ }^{14}$ and is thus unavoidable. It makes this CG model less-suitable for biomolecular systems.

Fig. 6 shows the bond-angle $\theta$ and torsional-angle $\varphi$ distributions for the simulations of pure hexadecane (NPT) at constant temperature and pressure. Different combinations of cutoff radius and time step do not influence the distributions significantly. The corresponding end-to-end distance distributions are shown in Fig. 7. Again the different combinations of 


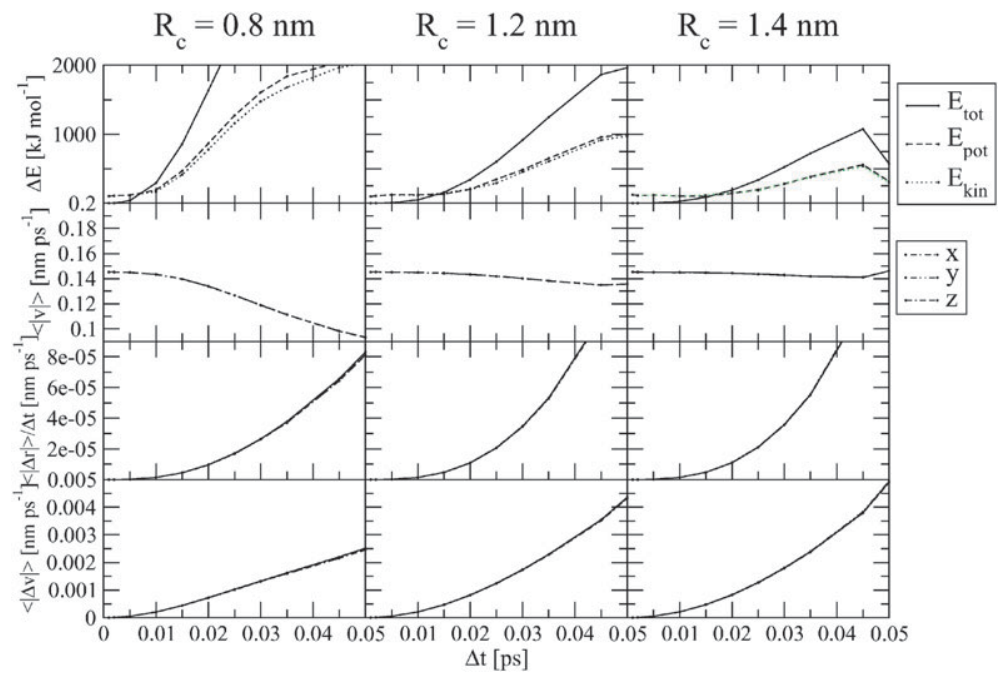

Fig. 1 Energy fluctuations (total $E_{\text {tot }}$ solid lines, potential $E_{\text {pot }}$ dashed lines, kinetic $E_{\text {kin }}$ dotted lines) and higher-order leap-frog terms ( $x$-, $y$-, $z$-components) calculated over the final $630 \mathrm{ps}$ of the $1260 \mathrm{ps}$ simulations of liquid water at NVE as a function of the cutoff radius $R_{\mathrm{c}}$ and time step $\Delta t$. The three colums represent the different cutoff radii $R_{\mathrm{c}} 0.8 \mathrm{~nm}, 1.2 \mathrm{~nm}$, and $1.4 \mathrm{~nm}$. First row: energy fluctuations, second row: averaged absolute velocities, $\langle|v|\rangle$, third row: averaged absolute third-order term (eqn (3)) for positions divided by $\Delta t$, fourth row: averaged absolute third-order term (eqn (4)) for velocities.

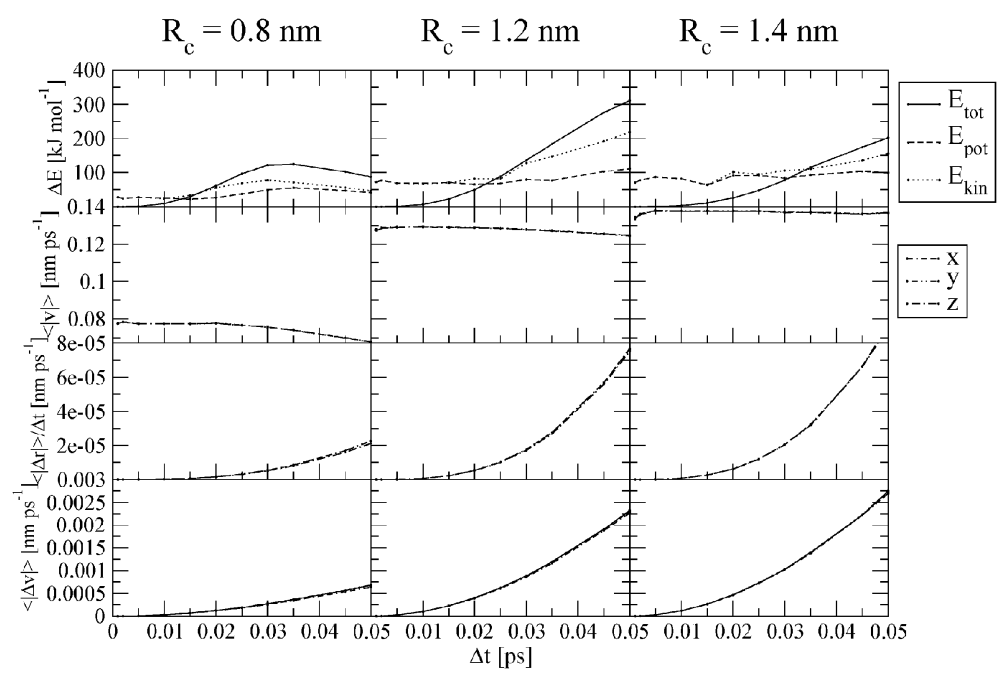

Fig. 2 Energy fluctuations and higher order leap-frog terms calculated over the final $630 \mathrm{ps}$ of the 1260 ps simulations of liquid hexadecane at NVE as a function of the cutoff radius $R_{\mathrm{c}}$ and time step $\Delta t$. The three columns represent the different cutoff radii $R_{\mathrm{c}} 0.8 \mathrm{~nm}, 1.2 \mathrm{~nm}$ and $1.4 \mathrm{~nm}$. First row: energy fluctuations, second row: averaged absolute velocities, $\langle|v|\rangle$, third row: averaged absolute third-order term (eqn (3)) for positions divided by $\Delta t$, fourth row: averaged absolute third-order term (eqn (4)) for velocities.

$R_{\mathrm{c}}$ and $\Delta t$ do not influence the behaviour of the system significantly.

In Table 3 , the free enthalpy of solvation $\left(\Delta G_{\text {solv }}\right)$ of one $\mathrm{CG}$ hexadecane molecule in a solution of 1192 CG water molecules is shown. The values do not vary significantly with $R_{\mathrm{c}}$ and $\Delta t$.

Table 3 also illustrates the distortive effect that may arise when a system that consists of two components that do not readily exchange kinetic energy and that are each subject to a different sink or source of energy, is coupled to only one temperature bath instead of to two separate baths, i.e. one for each component. The temperature of the system is related to the temperature of the components, e.g. solute and solvent, by the relation

$$
T_{\text {system }}=f_{\text {solute }} T_{\text {solute }}+f_{\text {solvent }} T_{\text {solvent }}
$$

where $f_{\text {solute }}$ is the fraction of solute degrees of freedom and $f_{\text {solvent }}$ the fraction of solvent degrees of freedom in the system. If the system is coupled to one temperature bath of temperature $T_{\text {ref }}$ and the solute picks up more energy than the solvent, e.g. due to integration errors or cut-off noise, we will find that

$$
T_{\text {solute }}<T_{\text {system }} \approx T_{\text {ref }}<T_{\text {solute }}
$$




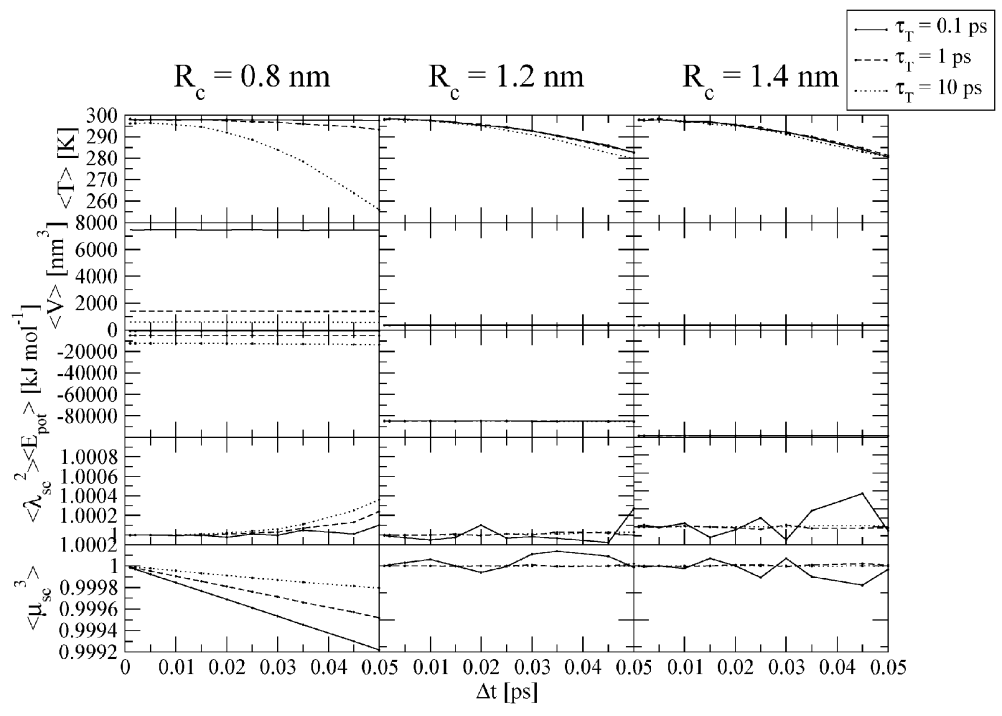

Fig. 3 Average temperature $T$, volume $V$, potential energy $E_{\text {pot }}$, squared temperature scaling factor $\lambda_{\text {sc }}$ (eqn (9)), and cubed pressure scaling factor $\mu_{\mathrm{sc}}$ (eqn (11)) calculated over the final $630 \mathrm{ps}$ of the $1260 \mathrm{ps}$ simulations of liquid water at NPT as a function of the cutoff radius $R_{\mathrm{c}}$ and time step $\Delta t$. The temperature was held constant using weak coupling with $\tau_{T}=0.1 \mathrm{ps}$ (solid lines), $1 \mathrm{ps}$ (dashed lined), and $10 \mathrm{ps}$ (dotted lines). The three columns represent the different cut-off radii $R_{\mathrm{c}}=0.8 \mathrm{~nm}, 1.2 \mathrm{~nm}$ and $1.4 \mathrm{~nm}$.

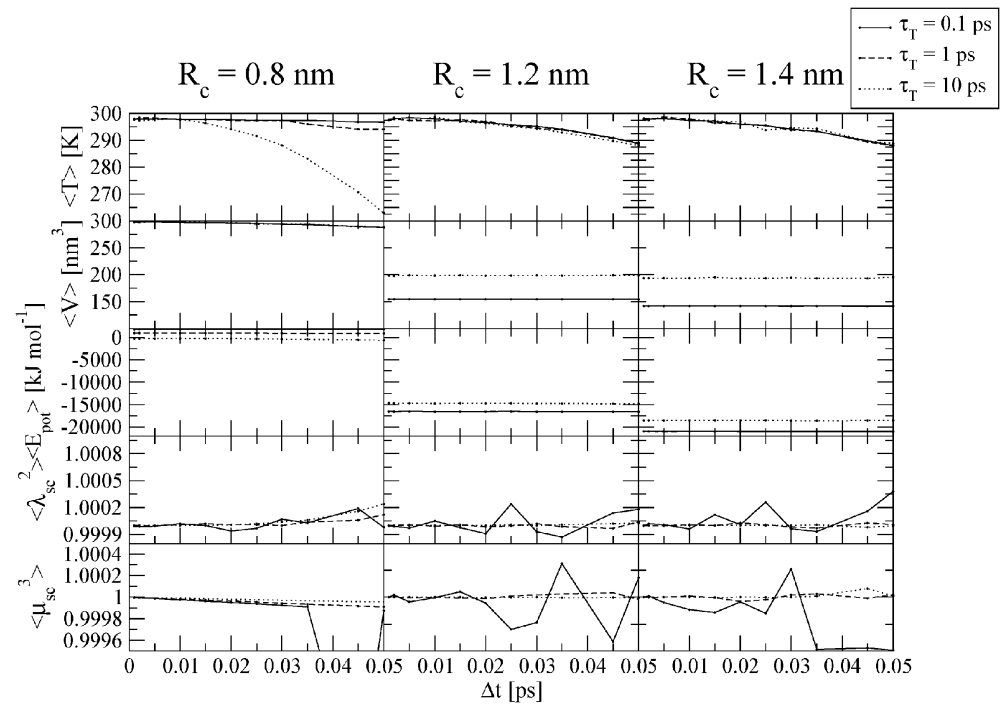

Fig. 4 Average temperature $T$, volume $V$, potential energy $E_{\text {pot }}$, squared temperature scaling factor $\lambda_{\text {sc }}$ (eqn (9)), and cubed pressure scaling factor $\mu_{\mathrm{sc}}$ (eqn (11)) calculated over the final $630 \mathrm{ps}$ of the $1260 \mathrm{ps}$ simulations of liquid hexadecane at NPT as a function of the cutoff radius $R_{\mathrm{c}}$ and time step $\Delta t$. The temperature was held constant using weak coupling with $\tau_{T}=0.1 \mathrm{ps}$ (solid lines), $1 \mathrm{ps}$ (dashed lined) and $10 \mathrm{ps}$ (dotted lines). The three columns represent the different cut-off radii $R_{\mathrm{c}} 0.8 \mathrm{~nm}, 1.2 \mathrm{~nm}$ and $1.4 \mathrm{~nm}$.

with

$$
\frac{T_{\text {solute }}-T_{\text {system }}}{T_{\text {solvent }}-T_{\text {system }}}=-\frac{f_{\text {solvent }}}{f_{\text {solute }}} .
$$

If the solvent picks up more energy than the solute, the roles of solute and solvent in eqn (13) and (14) are exchanged. In all-atom simulations of proteins in water, the latter is the case, because the $\mathrm{H}_{2} \mathrm{O}$ molecules are generally more prone to cut-off noise than the relatively less mobile protein and the protein bond lengths are generally constrained. For the CG hexane in CG water simulation reported in Table 3, the opposite is the case: the single-particle Lennard-Jones water is less prone to finite integration time step noise than the CG hexadecane molecule with its bond-length and bond-angle vibrations, leading to an artificial cooling of the solvent and heating of the solute. This effect disappears when each component is separately coupled to a temperature bath.

\section{Discussion}

Coarse-grained (CG) models have become more-and-more popular in recent years and thus it has become more important that these are correctly parametrised. The results reported here suggest a reparametrisation of one very basic feature of the CG model of Marrink et al. ${ }^{10,14,15}$ the time step that is used to integrate the Newtonian equations of motion. A time step of 
Table 2 Physical-chemical properties of pure CG water and pure CG hexadecane using NPT $\left(\tau_{T}=0.1 \mathrm{ps}, \tau_{p}=0.5 \mathrm{ps}\right)$ and excess free energies using NVT $\left(\tau_{T}=0.1 \mathrm{ps}\right)$ thermodynamic boundary conditions. The NPT data are averaged over the final $630 \mathrm{ps}$ of the $1890 \mathrm{ps}$ long simulations

\begin{tabular}{|c|c|c|c|c|c|c|c|c|}
\hline & $R_{\mathrm{c}} / \mathrm{nm}$ & $\Delta t[\mathrm{fs}]$ & $\begin{array}{l}\mathrm{NPT} \\
T / \mathrm{K}\end{array}$ & $\begin{array}{l}\mathrm{NPT} \\
\rho / \mathrm{kg} \mathrm{m}^{-3}\end{array}$ & $\begin{array}{l}\mathrm{NPT} \\
E_{\mathrm{pot}} / \mathrm{kJ} \mathrm{mol}^{-1}\end{array}$ & $\begin{array}{l}\mathrm{NPT} \\
D / \mathrm{cm}^{2} \mathrm{~s}^{-1}\end{array}$ & $\begin{array}{l}\mathrm{NPT} \\
S_{\text {conf }} / \mathrm{kJ} \mathrm{mol}^{-1} \mathrm{~K}^{-1}\end{array}$ & $\begin{array}{l}\mathrm{NVT} \\
\Delta F_{\text {exc }} / \mathrm{kJ} \mathrm{mol}^{-1}\end{array}$ \\
\hline \multirow[t]{6}{*}{ Water: } & 1.4 & 5 & 298 & 1102 & -98228 & $2.6 \times 10^{-8}$ & - & 22.1 \\
\hline & 1.4 & 10 & 297 & 1102 & -98245 & $1.9 \times 10^{-8}$ & - & 22.1 \\
\hline & 1.4 & 50 & 281 & 1093 & -97213 & $2.6 \times 10^{-8}$ & - & 18.1 \\
\hline & 1.2 & 10 & 298 & 1066 & -84747 & $6.5 \times 10^{-6}$ & - & 21.4 \\
\hline & 1.2 & 20 & 295 & 1067 & -84712 & $7.4 \times 10^{-7}$ & - & 18.3 \\
\hline & 1.2 & 50 & 282 & 1069 & -84927 & $6.7 \times 10^{-7}$ & - & 15.2 \\
\hline \multirow[t]{6}{*}{ Hexadecane: } & 1.4 & 5 & 298 & 1156 & -20971 & $1.3 \times 10^{-5}$ & 171 & 29.8 \\
\hline & 1.4 & 10 & 298 & 1156 & -20952 & $1.1 \times 10^{-5}$ & 172 & 30.1 \\
\hline & 1.4 & 50 & 288 & 1157 & -20983 & $3.5 \times 10^{-5}$ & 174 & 30.1 \\
\hline & 1.2 & 10 & 298 & 1064 & -16530 & $5.8 \times 10^{-5}$ & 173 & 30.3 \\
\hline & 1.2 & 20 & 297 & 1063 & -16543 & $1.8 \times 10^{-5}$ & 171 & 31.2 \\
\hline & 1.2 & 50 & 289 & 1063 & -16559 & $1.6 \times 10^{-5}$ & 173 & 30.9 \\
\hline
\end{tabular}

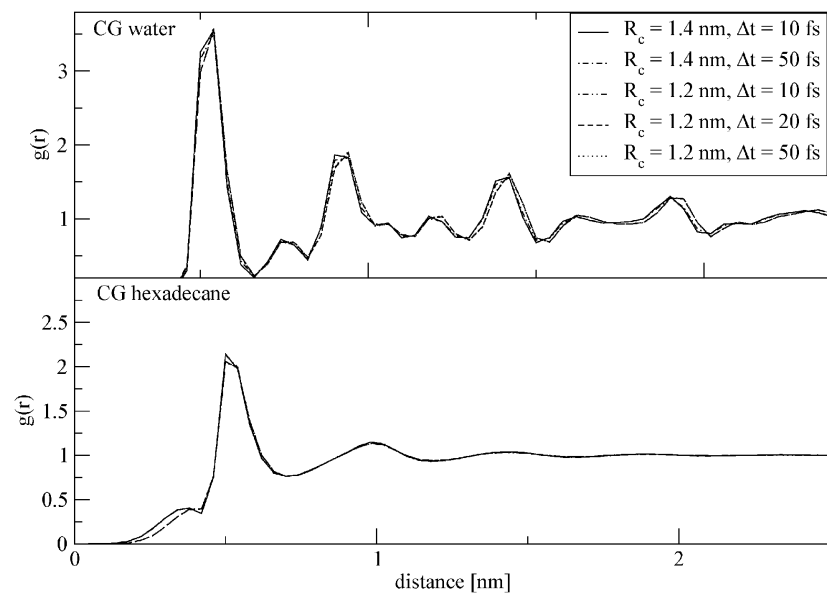

Fig. 5 Radial distribution functions $g(r)$ calculated from the final $630 \mathrm{ps}$ of the $1890 \mathrm{ps}$ simulations of liquid water and liquid hexadecane at NPT for different combinations of the cutoff radius $R_{\mathrm{c}}$ and time step $\Delta t$.

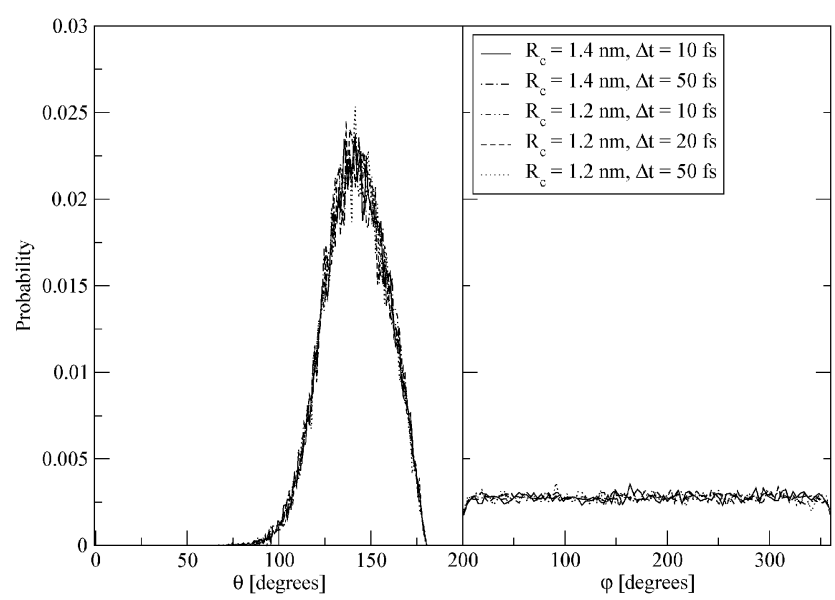

Fig. 6 Bond-angle $\theta$ distributions and torsional-angle $\varphi$ distributions calculated from the final 630 ps of the 1890 ps simulations of hexadecane at NPT for different combinations of the cut-off radius $R_{\mathrm{c}}$ and time step $\Delta t$.

$\Delta t=50 \mathrm{fs}$, that has been suggested and used by Marrink et al. ${ }^{10,14}$ is considered too large, since at NVE conditions the total energy fluctuations are significantly larger than the

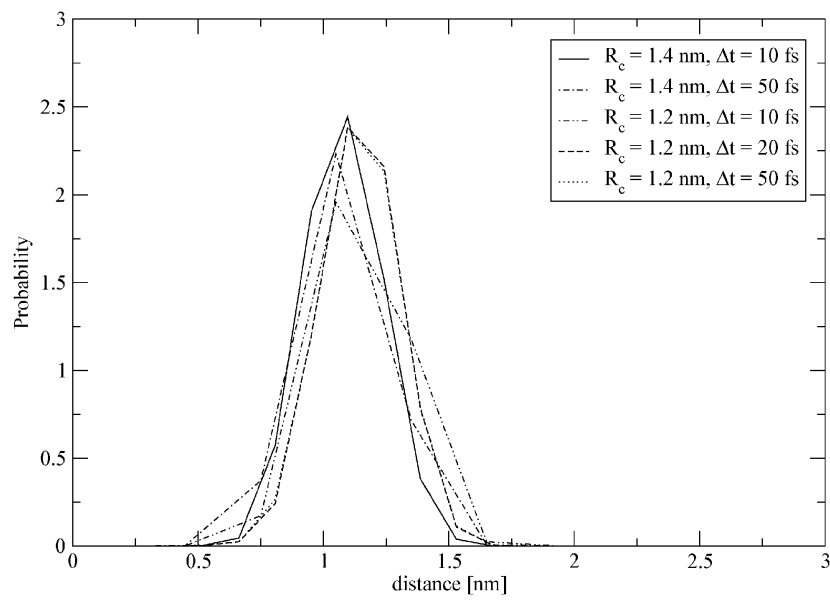

Fig. 7 Distribution of end-to-end of chain distances calculated from the final $630 \mathrm{ps}$ of the $1890 \mathrm{ps}$ simulations of liquid hexadecane at NPT for different combinations of the cut-off radius $R_{\mathrm{c}}$ and time step $\Delta t$.

fluctuations in the kinetic and potential energies. We note that the use of a shifting function masks the errors introduced by the use of a cutoff distance for non-bonded interactions. The ratio of total energy fluctuations over kinetic energy fluctuations becomes smaller, but the physical model does not become a better approximation of the real molecular system. Physicalchemical properties are affected by the use of a long time step, most strikingly the average temperature of the system, that is lowered by $20 \mathrm{~K}$ in the case of using time steps of $50 \mathrm{fs}$.

Our results show that for a cutoff radius of $R_{\mathrm{c}}=1.4 \mathrm{~nm}$ a maximum of time step $\Delta t=10 \mathrm{fs}$ should be used in coarsegrained model simulations based on Lennard-Jones interaction functions in order to avoid energy flow in or out of the system.

Analysis of the CG water model of ref. 10, 14 and 15 shows that the Lennard-Jones parameters are such that at physiological temperatures the model is well into the solid region of the phase diagram, making it less suitable for use in biomolecular simulations.

\section{Acknowledgements}

Financial support was obtained from the Swiss National Science Foundation (M.G.G.), and through the National 
Center of Competence in Research (NCCR) Structural Biology of the Swiss National Science Foundation, which is gratefully acknowledged.

\section{References}

1 M. Müller, K. Katsov and M. Schick, Biological and synthetic membranes: What can be learned from a coarse-grained description? Phys. Rep., 2006, 434, 113-176.

2 Gregory A. Voth, Coarse-Graining of Condensed Phase and Biomolecular Systems, CRC Press, Florida, USA, 2008.

3 B. Smit, P. A. J. Hilbers, K. Esselink, L. A. M. Rupert, N. M. van Os and A. G. Schlijper, Computer simulations of a water/oil interface in the presence of micelles, Nature, 1990, 348, 624-625.

4 R. Goetz and R. Lipowsky, Computer simulations of bilayer membranes: Self-assembly and interfacial tension, J. Chem. Phys., 1998, 108, 7397-7409.

5 R. D. Groot, T. J. Madden and D. J. J. Tildesley, On the role of hydrodynamic interactions in block copolymer microphase separation, J. Chem. Phys., 1999, 110, 9739-9749.

6 J. C. Shelley, M. Y. Shelley, R. C. Reeder, S. Bandyopadhyay and M. L. Klein, A coarse grain model for phospholipid simulations, J. Phys. Chem. B, 2001, 105, 4464-4470.

7 S. O. Nielsen, C. F. Lopez, G. Srinivas and M. L. Klein, A coarse grain model for n-alkanes parameterized from surface tension data, J. Chem. Phys., 2003, 119, 7043-7049.

8 K. Kremer, Computer simulations for macromolecular science, Macromol. Chem. Phys., 2003, 204, 257-264.

9 C. C. Liew and M. Mikami, A coarse-grained model for particle dynamics simulations of complex fluids, Chem. Phys. Lett., 2003, 368, 346-351.

10 S. J. Marrink, A. H. de Vries and A. E. Mark, Coarse grained model for semiquantitative lipid simulations, J. Phys. Chem. B, 2004, 108, 750-760.

11 E. S. Boek, T. J. Padding, W. K. den Otter and W. J. Briels, Mechanical properties of surfactant bilayer membranes from atomistic and coarse-grained molecular dynamics simulations, J. Phys. Chem. B, 2005, 109, 19851-19858.

12 A. Y. Shih, A. Arkhipov, P. L. Freddolino and K. Schulten, Coarse grained protein-lipid model with application to lipoprotein particles, J. Phys. Chem. B, 2006, 110, 3674-3684.

13 W. Shinoda, R. DeVane and M. L. Klein, Multi-property fitting and parameterization of a coarse grained model for aqueous surfactants, Mol. Simul., 2007, 33, 27-36.

14 S. J. Marrink, H. J. Risselada, S. Yefimov, D. P. Tieleman and A. H. de Vries, The MARTINI force field: Coarse grained model for biomolecular simulations, J. Phys. Chem. B, 2007, 111, $7812-7824$.

15 L. Monticelli, S. K. Kandasamy, X. Periole, R. G. Larson, D. P. Tieleman and S. J. Marrink, The MARTINI coarse-grained force field: Extension to proteins, J. Chem. Theory Comput., 2008, 4, 819-834.

16 Y. C. Kim and G. Hummer, Coarse-grained models for simulations of multiprotein complexes: Application to ubiquitin binding, J. Mol. Biol., 2008, 375, 1416-1433.

17 W. G. Noid, J.-W. Chu, G. S. Ayton, V. Krishna, S. Izvekov, G. A. Voth, A. Das and H. C. Andersen, The multiscale coarsegraining method. I. a rigorous bridge between atomistic and coarse-grained models, J. Chem. Phys., 2008, 128, 244114.

18 W. G. Noid, P. Liu, Y. Wang, J.-W. Chu, G. S. Ayton, S. Izvekov, H. C. Andersen and G. A. Voth, The multiscale coarse-graining method. II. numerical implementation for coarse-grained molecular models, J. Chem. Phys., 2008, 128, 244115.

19 P. J. Bond and M. S. P. Sansom, Insertion and assembly of membrane proteins via simulation, J. Am. Chem. Soc., 2006, 128, $2697-2704$.

20 P. J. Bond, J. Holyoake, A. Ivetac, S. Khalid and M. S. P. Sansom, Coarse-grained molecular dynamics simulations of membrane proteins and peptides, J. Struct. Biol., 2007, 157, 593-605.

21 W. F. van Gunsteren and H. J. C. Berendsen, Algorithms for macromolecular dynamics and constraint dynamics, Mol. Phys., 1977, 34, 1311-1327. 
22 H. J. C. Berendsen and W. F. van Gunsteren, Molecular dynamics simulations: Techniques and approaches, in Molecular Liquids Dynamics and Interactions, ed. A. J. Barnes, W. J. Orville-Thomas and J. Yarwood, Reidel, Dordrecht, 1984, pp. 475-500.

23 H. J. C. Berendsen and W. F. van Gunsteren, Practical algorithms for dynamic simulations, in Molecular-Dynamics Simulation of Statistical-Mechanical Systems, ed. G. Ciccotti and W. G. Hoover, North-Holland, 1986, volume course 97, pp. 43-65.

24 D. van der Spoel, E. Lindahl, B. Hess, G. Groenhof, A. E. Mark and H. J. C. Berendsen, GROMACS: fast, flexible, and free, J. Comput. Chem., 2005, 26, 1701-1718.

25 R. Baron, D. Trzesniak, A. H. de Vries, A. Elsener, S. J. Marrink and W. F. van Gunsteren, Comparison of thermodynamic properties of coarse-grained and atomic-level simulation models, ChemPhysChem, 2007, 8, 452-461.

26 M. Christen, P. H. Hünenberger, D. Bakowies, R. Baron, R. Bürgi, D. P. Geerke, T. N. Heinz, M. A. Kastenholz, V. Kräutler, C. Oostenbrink, C. Peter, D. Trzesniak and W. F. van Gunsteren, The GROMOS software for biomolecular simulation: GROMOS05, J. Comput. Chem., 2005, 26, 1719-1751.

27 M. A. Cuendet and W. F. van Gunsteren, On the calculation of velocity-dependent properties in molecular dynamics simulations using the leapfrog integration algorithm, J. Chem. Phys., 2007, 127, 184102.
28 H. J. C. Berendsen, J. P. M. Postma, W. F. van Gunsteren, A. DiNola and J. R. Haak, Molecular dynamics with coupling to an external bath, J. Chem. Phys., 1984, 81, 3684-3690.

29 J.-P. Ryckaert, G. Ciccotti and H. J. C. Berendsen, Numerical integration of the Cartesian equations of motion of a system with constraints: Molecular dynamics of $n$-alkanes, J. Comput. Phys., 1977, 23, 327-341.

30 T. Huber, A. E. Torda and W. F. van Gunsteren, Local elevation-a method for improving the searching properties of moleculardynamics simulation, J. Comput.-Aided Mol. Des., 1994, 8, 695-708.

31 A. P. Nanzer, W. F. van Gunsteren and A. E. Torda, Parametrisation of time-averaged distance restraints in MD simulations, J. Biomol. Nucl. Magn. Reson., 1995, 6, 313-320.

32 R. Baron, A. H. de Vries, P. H. Hünenberger and W. F. van Gunsteren, Comparison of atomic-level and coarse-grained models for liquid hydrocarbons from molecular dynamics configurational entropy estimates, J. Phys. Chem. B, 2006, 110, $8464-8473$.

33 J. J. Nicolas, K. E. Gubbins, W. B. Streett and D. J. Tildesley, Equation of state for the Lennard-Jones fluid, Mol. Phys., 1979, 37, 1429-1454. 